\title{
Study on Primary Percutaneous Coronary Intervention (PCI) in Patient with Acute Myocardial Infarction: in-hospital and 30-days Survival Outcome
}

\author{
AQM Reza, AHMW Islam, S Munwar, S Talukder \\ Department of Invasive and Interventional Cardiology, Apollo Hospitals, Dhaka.
}

\begin{abstract}
:
Keywords:

Primary PCI,

STEMI.

Objective: Aim of our study was to evaluate the safety and survival outcome of Primary PCI (pPCI) in patients with Acute Myocardial infarction in our hospital setting.

Methods: Total 30 (Female 5; Male 25) patients were enrolled in this study who were brought in to our hospital with STEMI. Average age was, Male 56, Female 52. Primary PCI was performed after transferring patient from Emergency Department (ED) to Cardiac Catheterization laboratory. Cardiovascular risk factors among the studied population were Dyslipidemia, Diabetes Mellitus, Hypertension, Smoking and Family History.

Results: Primary PCI either with Bare Metal Stent (BMS) or Drug Eluting Stent (Sirolimus-eluting stent or Paclitaxel stent) were performed in total 13 LAD lesion and 15 RCA lesion and 2 LCX lesion. BMS used were $66.7 \%$, Sirolimus $20 \%$ and Paclitaxel-eluting stent $13.3 \%$. Total 2 patients expired but due to hemorrhagic CVA and refractory heart failure. At presentation, ECG evidenced diagnosis were Acute Anterior Wall MI : 12 (40\%), Inferior MI: 16 (53\%), Infero-Posterior MI: 2 (6.7\%). Our study showed that Primary PCI increases the higher survival outcome 28 (93.3\%) out of 30 patients with acute $M I$.

Conclusion: Our present study revealed that revascularization by Primary PCI showed safety and better percentage of In-hospital and 30-days survival outcome in patients with Acute myocardial infarction in our hospital setting.
\end{abstract}

(Cardiovasc. j. 2010; 2(2) : 212-217)

\section{Introduction:}

Primary Percutaneous coronary intervention (pPCI) is considered to be superior to thrombolytic treatment for ST elevation acute myocardial infarction (STEMI) especially, in a hospital with angioplasty facilities. ${ }^{1-3}$

It has been established by several investigators that the achievement of useful means of successful reperfusion was superior in $\mathrm{pPCI}$ than compared with thrombolytic therapy. ${ }^{4-5}$ Door to balloon time,${ }^{6-7}$ is an important key factor in the success of pPCI. Many have demonstrated that patients, who had pPCI within 2 hrs of symptoms onset had lower mortality and greater myocardial salvage after pPCI and higher rates of complete reperfusion. ${ }^{8}$ Aim of our study was to evaluate safety and the survival out come and benefits of pPCI in our hospital.

\section{Patient Population:}

Patients were randomized from the cases who presented to our ED with the onset of chest pain outside hospital of any age for at least 30 minutes but less than $12 \mathrm{hrs}$ in duration. ECG criteria were associated with ST elevation at least $0.1 \mathrm{mV}$ in 2 or more ECG leads who underwent pPCI at this hospital. Prior to pPCI, informed written consent were taken from the patient or guardians.

\section{Methods:}

Patients were treated with the loading doses of $300 \mathrm{mg}$ Aspirin and 600mg Clopidegrol in hospital emergency. With Diagnostic Coronary angiography was done before Percutaneous Coronary Intervention (PCI) to locate culprit infarct related territory or artery. After thrombus suction from the occluded site by a thrombuster sucker if

Address of Correspondence- Dr A H M Waliul Islam, Department of Invasive and Interventional Cardiology, Apollo Hospitals Dhaka, Bangladesh. 
indicated, balloon dilatations done in all cases, followed by stenting in the culprit lesion. Further smoothening was done by post-dilatation whenever required. Successful PCI was defined as a visually assessed 20-30\% residual stenosis with TIMI III distal flow. IVUS was not available. Before PCI, IV heparin bolus of 7000 IU was given. Platelet glycoprotein IIb/IIIa-receptor blockers were administered at the discretion of the physicians. All patients were routinely underwent 12-lead ECG before and after the PCI to detect the procedure related ischaemic changes and or the presence of new pathologic $Q$ waves. Blood sample for Cardiac CK-MB was measured at arrival into our hospital.

\section{Primary Survival outcomes or end-points:}

The predicted endpoints were 30-day all-cause mortality and re-infarction or disabling stroke or LVEF $<30 \%$. Clinical reinfarction was diagnosed as any new infarction occurring after index infarct and unrelated to $\mathrm{PCI}$ or $\mathrm{CABG}$ with raised CK-MB above its previous nadir and history of chest discomfort or ECG changes.

Statistical analysis -Data were presented as Mean $\pm \mathrm{SD}$ and percentage.

\section{Results:}

Primary PCI were performed in total 30 patients (Male 25, Female 5). Table I Shows the profile and clinical data of studied population. Male are older than female. Both systolic and Diastolic BP was higher in female than male. Cardiac CK-MB was higher in male than female. No differences were observed on average no of CAD risk factors in either group. Both SBP and DBP (recorded on arrival to ER) were higher in female than compared to male $(126.7 \pm 40.4$ V.S $125.0 \pm 26.0 ; 80 \pm 26.5$ V.S $77.7 \pm 13.9)$. Cardiac CK-MB were raised in both groups (Male: $57.2 \pm 65.3$, Female 77.9 \pm 76.0 ). Door-to-balloon time in female was: $58.5 \pm 29.8$ versus male: $125.7 \pm 54.3$. The distribution of Cardiovascular risk factors were Dyslipidemia 26 (86.7\%), Hypertension 19 (63.3\%), Diabetes Mellitus 12 (40\%) and smoking 11 (36.7\%)of all male patient in the studied population.

PCI to LAD in 13 patients (43.3\%), PCI to RCA in 15 patients (50\%) and PCI to LCX in 2 patient (6.7\%) were done (Table II).

Average stent size required was almost same in both LAD, LCX and RCA. ECG evidenced diagnosis on arrival at ED with the Anterior MI 12 (40\%), Inferior MI 16 (53\%), Infero-post MI 2 (6.7\%) respectively (Table III). Total 2 patients with Anterior MI had subsequent cardiogenic shock due to their disease ailment.

Table-I

Demographic profile and clinical data of patients.

\begin{tabular}{lcc}
\hline & Male & Female \\
\hline Number & $25(75 \%)$ & $5(25 \%)$ \\
Age (yrs) & $56.0 \pm 9.7$ & $52.0 \pm 9.1$ \\
SBP(mmHg) & $125.0 \pm 26.0$ & $126.7 \pm 40.4$ \\
DBP(mmHg) & $77.7 \pm 13.9$ & $80.0 \pm 26.5$ \\
CK-MB (U/L) & $57.2 \pm 65.3$ & $77.9 \pm 76.0$ \\
Trop-I (U/L) & $6.74 \pm 16.6$ & $7.4 \pm 12.4$ \\
No of CAD Risk Factor & $2.6 \pm 1.0$ & $2.5 \pm 0.6$ \\
Door-to-Balloon Time (Min) & $125.7 \pm 54.3$ & $58.5 \pm 29.8$ \\
\hline
\end{tabular}

Data were presented as Mean \pm SD

Table-II

Average size of stents used with inflation pressure

\begin{tabular}{lcccc}
\hline & $\mathrm{No}(\%)$ & Length $(\mathrm{mm})$ & Diameter $(\mathrm{mm})$ & Inflation Pressure(ATM) \\
\hline LAD & $13(43.3 \%)$ & $21.5 \pm 7.3$ & $3.2 \pm 0.5$ & $15.1 \pm 4.1$ \\
LCX & $2(6.7 \%)$ & $23 \pm 5.0$ & $3.0 \pm 0.2$ & $16 \pm 1.4$ \\
RCA & $15(50 \%)$ & $21.7 \pm 0.6$ & $3.5 \pm 0.3$ & $15.5 \pm 2.3$ \\
\hline
\end{tabular}

Data were presented as Mean $\pm \mathrm{SD}$ 
Table III

Mode of presentation in our hospital

\begin{tabular}{|c|c|c|c|}
\hline & & Cardiogenic Shock & IABP \\
\hline nt MI & $12(40 \%)$ & $2(6.7 \%)$ & \\
\hline Ac. Inferior MI & $16(53 \%)$ & & $(11.7 \%)$ \\
\hline Ac. Inf.-Post MI & $2(6.7 \%)$ & & \\
\hline
\end{tabular}

BMS was used in $66.7 \%$ cases followed by Sirolimus Eluting stent in 205 patients and Paclitaxel eluting stent in $13.3 \%$ patients (Fig 1).
Total in-hospital and 30-days survival outcome were 28 (93.3\%). Total $2(6.7 \%)$ patient expired because of CVA with intracerebral hemorrhage in 1 patients and 1patient due to pump failure. All 28 (93.3\%) those survived after Primary PCI were discharged in a stable haemodynamic condition and being followed-up at an interval of 1 month, 6 month and 9 month time in our Cardiac OPD.

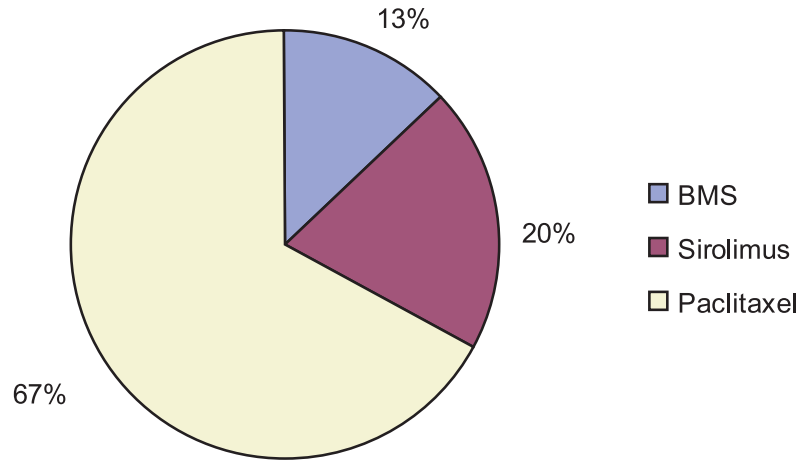

Fig 1: Pie graph showing the percentage of stent used .
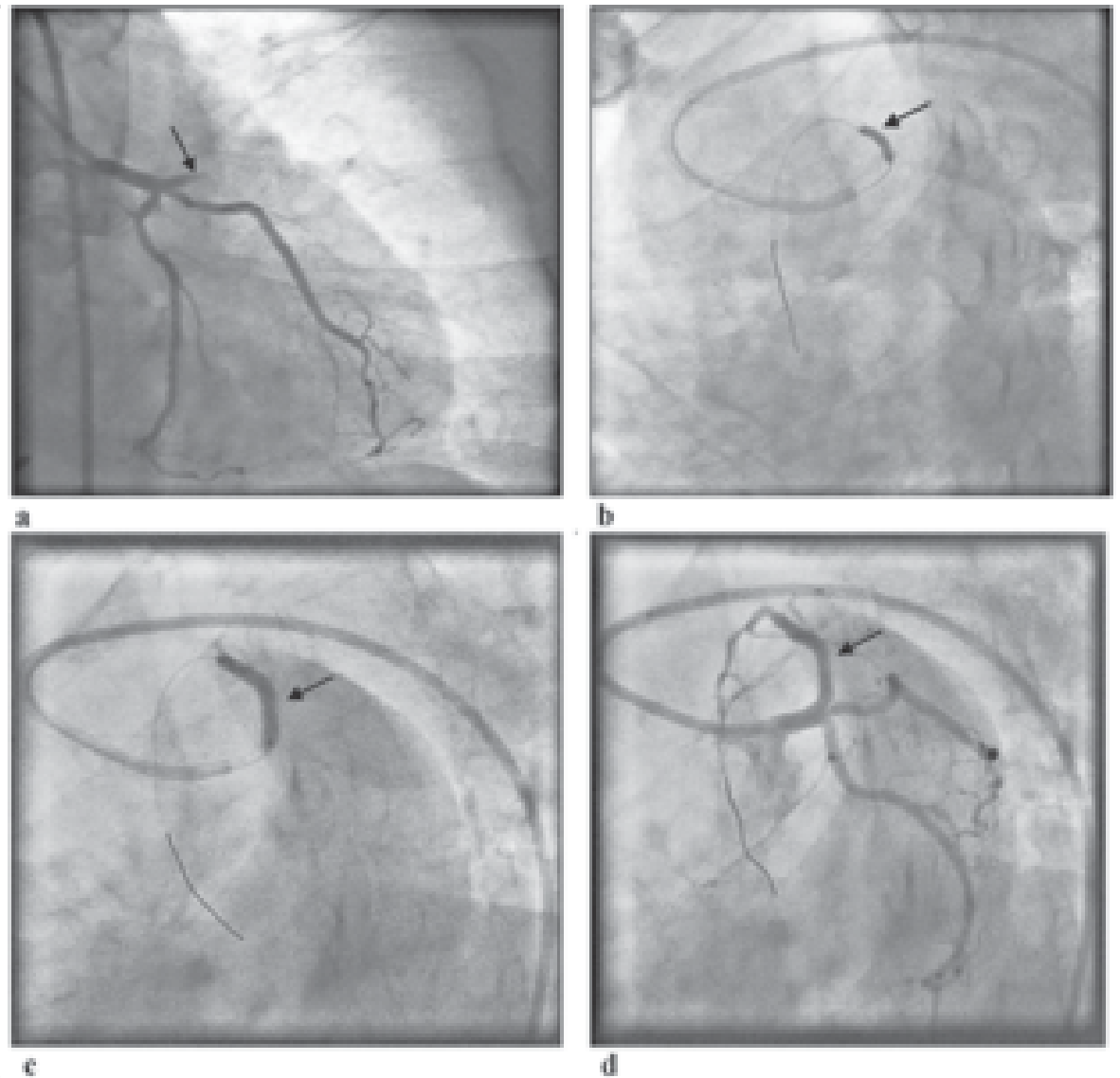

Fig.2 Primary PCI in Proximal LAD in a patient with acute Anterior Myocardial Infarction. 2a. Occluded proximal LAD (arrow), 2b: Ballooning (arrow), 2c: Stenting,2d: Post PCI TIMI III distal flow. 

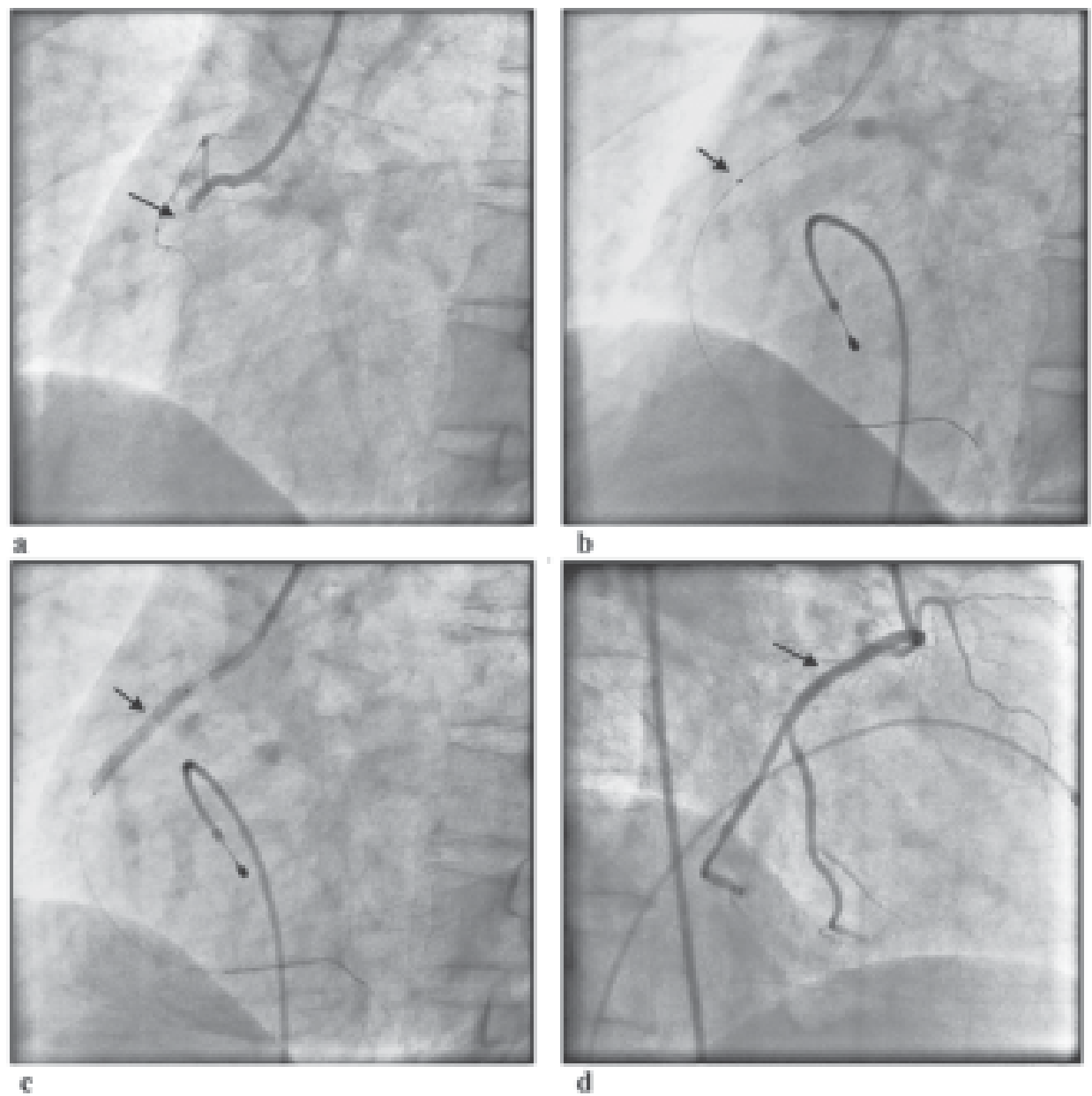

Fig.-3: Primary PCI in Proximal RCA in a patient with acute Inferior Myocardial Infarction. 3a. Occluded proximal RCA (arrow), 3b: Thrombus suction (arrow), 3c: Stenting, 3d: Post PCI TIMI III distal flow

\section{Discussion:}

The advent of thrombolytic therapy in the treatment of AMI patient in late 1980s improved survival outcome, recurrent ischaemic events continued to occur unpredictably in the post MI period. Later, invention of PTCA and PCI, has improved a lot in the stent era. Advances in stent implantation technique derived from intravascular ultrasounds imaging ${ }^{9}$ and recognition of adequate plaque burden, ${ }^{10}$ the incidences of sub-acute stent thrombosis has fallen in complex subsets. ${ }^{11}$ With the comparison of PTCA, the implantation of stents in the elective setting has been shown to reduce angiographic restenosis and improve late clinical outcomes. ${ }^{12-13}$ survival outcome on pPCI has established that the benefits of pPCI in patients with acute myocardial infarction is superior to the befits of thrombolytic therapy. ${ }^{14,16}$

Stone et al, has documented that Primary stenting is safe, feasible in the majority of patients with acute myocardial infarction and results in excellent short-term outcomes. ${ }^{17}$

In our present study, we used mainly Bare metal stent (BMS) and Drug Eluting Stents (DES) Sirolimus and Paclitaxel-eluting stent. Although, the use of DES and its roll on acute stent thrombosis is still debatable, majority are using BMS. Our patient, treated with DES has no documented acute stent thrombosis.

Our present study had shown the increases in primary endpoint of $28(93.3 \%) .2$ patient died (1 patient had CVA and 1 patient refractory heart failure), does also implicating procedural success of pPCI. Although, lacking of IVUS facilities, we are unable to get optimal result. Visual $<20 \%$ residual stenosis was considered as successful pPCI in this study. None of the patient had procedure related complications like haematoma at puncture site, acute or 30 day stent thrombosis. ${ }^{18}$ 
Also, we found that female patients are developing $\mathrm{CAD}$ leading to $\mathrm{AMI}$ and undergone subsequent pPCI with better in-hospital and 30-days survival out come than male subset of our patient population. This was probably due to the lower (average 58 minutes) door-to-balloon time in female patient than compare to male patients (average 125 minutes). The possible explanation is, in case of female patient, husband and or family member's are the key person responsible to give consent at earliest time, thus reducing the door-to-balloon time. On the other hand, in case of male patient, the reason behind the delayed or prolonged doorto-balloon time was due to the delayed consent from patient himself, as he is the decision maker. Marked increase of Cardiac CK-MB in both male and female indicates increase area of myocardial tissue damage and may be an important predictor of survival.

The main benefits of pPCI over thrombolysis is the achievements of a higher rate of coronary recanalization with a lower risk of intracranial bleeding and Left ventricular free wall rupture. ${ }^{4,19-}$ ${ }^{20}$ Although, 1 patient had intracranial bleeding in post pPCI stay at hour hospital.

In patient undergoing pPCI, procedural success provides significant prognostic value. Procedural success, primary endpoint and survival out come of our present study is consistent with PAMI stent pilot trial. ${ }^{17}$ Several trials on facilitated primary angioplasty in whom early pharmacological reperfusion before pPCI may further improves outcomes in AMI. 14-15

\section{Study Limitation:}

Door-to-balloon time was exactly not possible because of lacking of expression of exact onset time and consent for pPCI either by the patient or attendants. Future needs are to record exact time of onset of chest pain and getting immediate consent on arrival at Emergency Department. Lack of availability of IVUS was also a drawback for us to see intravascular thrombus burden and exact stent implantation.

\section{Conclusion:}

We found that Primary PCI is safe and increase the in-hospital and 30-days survival outcome in patient with acute myocardial infarction. Our future perspective is to established the comparison of survival outcome in pPCI versus fibrinolytic therapy in our patient populations, reducing the door-to-balloon time below two hours, benefits of pPCI in NSTEMI the assessment of it's primary benefits.

\section{Conflict of interest- None.}

\section{References:}

1. Antman EM, Anbe DT, Armstrong PW, et al. ACC/AHA guide lines for the management of patient with STelevation Myocardial infarction; a report of the American college of cardiology/American heart Association task force on practice guidelines. J Am Coll Cardiol 2004;44:E1-211.

2. Anderson HR, Nielson TT, Rasmussen K, et al. A comparison of coronary angioplasty with fibrinolytic therapy in acute myocardial infarction. $N$ Eng $\mathrm{J} \mathrm{Med}$ 2003; 349:733-42.

3. Keely EC, Grines CL. Should patient with acute myocardial infarction be transferred to a tertiary center for primary angioplasty or receive it at qualified hospitals in the community? The case for emergency transfer for Primary Percutaneous coronary intervention. Circulation.2005;112:3520-32.

4. Grines CL, Browne KF, Marco J, et al. Primary angioplasty in myocardial infarction study group. A comparison of immediate angioplasty with thrombolytic therapy for acute nyocardial infarction. $N$ Eng J Med 1993;328:673-9.

5. Weaver WD, Simes RJ, Betriu A, et al. Comparison of primary coronary angioplasty and intravenous thrombolytic therapy for acute myocardial infarction: a qualitative review. JAMA 1997;278:2093-2098.

6. Canon CP, Antman EM, Walls R, Braunwald E. Time as an adjunctive agent to thrombolytic therapy. $J$ Thromb Thrombolysis.1994;1:27-34.

7. Canon CP, Gibson MC, Lambrew CT, et al. Relationship of symptom-onset-to-balloon time and Door-to-balloon time with mortality in patients undergoing angioplasty for acute myocardial infarction. JAMA 2000;283:22:2941-2947.

8. Bordie BR, Stuckey TD, Wall TC, et al. Importance of time to reperfuision for 30-day and late survival andc recovery of left bventricul;ar function after primary angioplasty for acute myocardial infarction. J Am Coll Cardiol 1998;32:1312-1319.

9. Moussa I, Di Mario, Francesco L. Et al. Subacute stent thrombosis and the anticoagulation controversy;changes in drug therapy, operator technique and the impact of intravascular ultrasound. Am J Cardiol 1996;78:Suppl 3A;13-7. 
10. Schomig A, Neuman FJ, Kastrati et al. A randomized comparison of antiplatelet and anticoagulation therapy after placement of coronary stents. $N$ Eng J Med 1996;334:1084-9.

11. Alfonso F, Rodriguez P, Phillips P, et al. Clinical and angiographic implications of coronary stenting in thrombus-containing lesion. J Am Coll Cardiol 1997;29:725-33.

12. Serruys PW, Jaegere P, Kiemeneji F, et al. A comparison of balloon expandable-stent implantation with balloon angioplasty in patients with coronary artery disease. $N$ Eng J Med 1994;331:489-495.

13. Fischman Dl, Leon MB, Baim DS, et al. A randomized comparison of coronary-stent placement and balloon angioplasty in the treatment of coronary artery disease. N Eng J Med 1994;331:496-501.

14. Bonnefoy E, Lapostolle F, Leizorovicz A, et al. Primary angioplasty versus prehospital finbrinolysis in acute myocardial infarction: a randomized study. Lancet 2002;360:825-9.

15. Ross AM, Coyne KS, Reiner JS, et al. A randomized trial comparing primary angioplasty with a strategy of short-acting thrombolysis and immediate planned rescue angioplasty in acute myocardial infarction:the PACT trial. J Am Coll Cardiol 1999;34:1954-1962.

16. Stone GW, Brodie BR, Griffin JJ, et al. Clinical and angiographic follow-up after primary stenting in acute myocardial infarction: PAMI stent pilot trial. Circulation 1999;99:1548-1554.

17. Stone GW, Brodie BR, Griffin JJ, et al. Prospective, Multicenter study of the safety and feasibility of primary stenting in acute myocardial infarction: In-Hospital and 30-day results of the PAMI Stent pilot Trial. J Am Coll Cardiol 1998;31:23-30.

18. Fox KA, Poole-Wilson PA, Henderson RA, et al. Interventional versus conservative treatment for patients with unstable angina or non-ST elevation MI; the British Heart Foundation RITA 3 randomized trial. Lancet 2002;360:743-51.

19. Moreno R, Lopez-Sendon J, Garcia E,et al. Primary angioplasty reduces the risk of left ventricular free wall rupture compared with acute myocardial infarction. $J$ Am Coll Cardiol 2002;39:598-603.

20. Garcia E, Elizaga J, Perez N, et al. Primary angioplasty vesrsus systemic thrombolysis in anterior myocardial infarction. J Am Coll Cardiol 1999;33:605-11. 